\title{
Effect of Doped Fiber Length on the Stretch Pulses of a Mode-Locked Erbium-Doped Fiber Laser ${ }^{1}$
}

\author{
S. J. Tan ${ }^{a}$, M. A. Ismail ${ }^{a}$, N. S. Shahabuddin ${ }^{a}$, H. Ahmad ${ }^{b}$, H. Arof ${ }^{a}$, and S. W. Harun ${ }^{a, b}, *$ \\ ${ }^{a}$ Department of Electrical Engineering, Faculty of Engineering, University of Malaya 50603 Kuala Lumpur, Malaysia \\ ${ }^{b}$ Photonics Research Center, University of Malaya, 50603 Kuala Lumpur, Malaysia \\ *e-mail: swharun@um.edu.my \\ Received January 27, 2012; in final form, February 9, 2012; published online June 12, 2012
}

\begin{abstract}
A passively self-starting mode-locked fiber ring laser is demonstrated using a highly concentrated Erbium doped fiber (EDF) with a saturable absorber. The effect of EDF length on the performance of the laser is investigated. Stable stretched pulses are obtained at wavelength region of $1560 \mathrm{~nm}$ with a repetition rate ranging from 10.1 to $12.1 \mathrm{MHz}$ and a pulse width stretching from 0.52 to $0.75 \mathrm{ps}$ as the EDF length is reduced from 4.5 to $1.5 \mathrm{~m}$. The repetition rate goes higher as the cavity length decreases when a shorter EDF is used but the pulse width reduces as the EDF length increases since the total group velocity dispersion (GVD) in the cavity is close to zero.
\end{abstract}

DOI: $10.1134 / \mathrm{S} 1054660 \mathrm{X} 12070134$

\section{INTRODUCTION}

Optical pulse fiber lasers are of great interest as they have been used in a wide range of applications depending on the wavelength and pulse width of the output for example in optical metrology laser surgery, optical signal processing and as a light source for optical communication [1-6]. Pulse fiber lasers are divided into two types which are Q-switching and mode locked fiber laser where pulses can be produced either actively or passively [7, 8]. Mode locked fiber lasers have gained much attention due to their capability of producing ultra-short pulses in picosecond and femtosecond regime. Moreover, they are very reliable, compact in size, and they produce high beam quality [9-13].

A variety of passive mode locking methods have been demonstrated using fast and slow saturable absorber, non-linear polarization rotation (NPR), addictive pulse mode locking, colliding mode locking and Kerr lens mode locking [14-19]. Unlike active mode locking, passive mode locking does not require an external modulator which is sychronized electronically to the cavity repetition rate. Instead, passive mode locking exploits optical nonlinear effect in material to initiate mode-locking. A saturable absorber (SA) is often used to self-start and achieve passive mode-locking. SAs are nonlinear optical elements which introduce certain optical loss depending on the intensity of the light beam incident upon them. If the incident light has low intensity, absorption will take place in the SA while high intensity light passes through the SA with minimum loss. In this paper, a passively mode-locked fiber ring laser is demonstrated using a highly concentrated Erbium doped fiber $(E D F)$ as the gain medium in conjunction with a

\footnotetext{
${ }^{1}$ The article is published in the original.
}

semiconductor SA. The effect of EDF length on the performance of the mode locked Erbium doped fiber laser (EDFL) is also investigated.

\section{EXPERIMENTAL SETUP}

The configuration of the proposed EDFL is shown in Fig. 1. It consists of a piece of EDF, wavelength division multiplexers (WDMs), an isolator, a polarization controller (PC), a saturable absorber (SA) and a $3 \mathrm{~dB}$ output coupler. The EDF has an erbium ion concentration of $2000 \mathrm{ppm}$, cut-off wavelength of $910 \mathrm{~nm}$, a pump absorption rate of $24 \mathrm{~dB} / \mathrm{m}$ at $980 \mathrm{~nm}$ and a

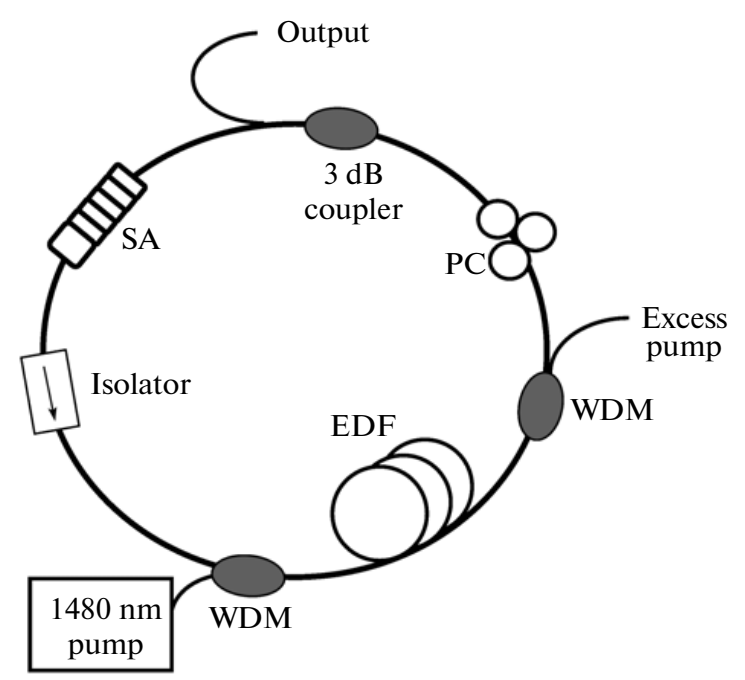

Fig. 1. Configuration of the proposed EDFL. 